\title{
Cubital Tunnel Syndrome Caused by a Ganglion Cyst Originating from the Ulnohumeral Joint: A Case Report
}

\author{
Jung-hoon $\mathrm{Kim}^{1}$, Woo-chul Cho ${ }^{1}$, Byung-chul Son ${ }^{1,2}$ \\ ${ }^{1}$ Department of Neurosurgery, Seoul St. Mary's Hospital, College of Medicine, The Catholic University of Korea, Seoul, \\ ${ }^{2}$ Catholic Neuroscience Institute, College of Medicine, The Catholic University of Korea, Seoul, Republic of Korea
}

Corresponding author:

Byung-chul Son

Department of Neurosurgery,

Seoul St. Mary's Hospital,

Catholic Neuroscience Institute,

College of Medicine, The

Catholic University of Korea,

222 Banpo-daero, Seocho-gu,

Seoul 06591, Republic of Korea

Tel: +82-2-2258-6122

Fax: +82-2-594-4248

E-mail: sbc@catholic.ac.kr

Received: August 16, 2018

Revised: August 19, 2018

Accepted: August 20, 2018
Ganglions are benign cysts bounded by dense connective tissue and filled with gelatinous fluid rich in hyaluronic acid and mucopolysaccharides. They occur most commonly in the knee and wrist. However, entrapment of the ulnar nerve by ganglion cysts has also been described to occur within the cubital tunnel. Despite of their incidence (3-8\%) among patients with cubital tunnel syndrome, they have been rarely reported. A 56-year-old male, professional golf instructor presented with a sudden onset of right hand paresthesia of 3-month duration. Paresthesia with decreased sensation in the lateral 2 digits and ulnar palm in his right hand and clumsiness in his right fingers were observed. Physical examination showed no weakness or atrophy of intrinsic hand muscles in his right hand. For preoperative diagnosis of ganglion cyst causing cubital tunnel syndrome, characteristic magnetic resonance imaging findings were quite helpful. On exploration, the ulnar nerve was found to be compressed by densely adherent, multilobular ganglion cyst within the cubital tunnel. A rare occurrence of ganglion cyst originating from medial ulnohumeral joint, compressing the ulnar nerve within the cubital tunnel, is experienced. Here we report an intraoperative findings for the ganglion cyst of medial ulnohumeral joint compressing the ulnar nerve. Early diagnosis of a ganglion cyst compressing the ulnar nerve within the cubital tunnel is essential to achieve successful treatment for this rare lesion.

Key Words: Cubital tunnel syndrome; Cysts; Elbow joint; Ganglion cysts; Ulnar nerve; Ulnar neuropathies

\section{INTRODUCTION}

Entrapment of the ulnar nerve is the second most common compression neuropathy in the upper extremity after carpal tunnel syndrome ${ }^{3,4)}$. Among the 5 potential sites for ulnar nerve entrapment, the cubital tunnel is the common site ${ }^{3,4,9)}$. Potential etiologies for ulnar nerve compression at the elbow are multiple and can include trauma, arthritis, heterotopic ossification, metabolic conditions predisposing to neuropathy, such as diabetes and alcoholism, external compression, soft tissue mass, and occupations that require repetitive or persistent elbow flexion ${ }^{4)}$.

A ganglion is a cystic, tumor-like lesion of unknown origin, which is surrounded by dense connective tissue filled with gelatinous fluid rich in hyaluronic acid and mucopolysaccharides $^{2,12)}$. Ganglion cysts are regarded as myxoid lesions, with suggested causes including synovial herniation and tissue degeneration or repeated trauma ${ }^{2,7,14}$. They are different from synovial cysts, a common cystic lesion around the joint, in absence of synovial cell lining in histologic examination and no communication with the joint cavity ${ }^{2,7,14}$. They can be cate- gorized according to their location to the joint: juxta-articular, intra-articular, and periosteal ${ }^{2}$. Symptomatic juxta-articular ganglion cysts are common in the knee and wrist ${ }^{2}$. However, medial elbow ganglion cysts, originating the ulnohumeral joints, causing cubital tunnel syndrome has already been reported $^{1,5,6,8,11,13)}$. Although their incidence among patients with cubital tunnel syndrome has been reported from $<3 \%$ to $8 \%$, they have been rarely reported ${ }^{6,13)}$. The authors report a rare occurrence of cubital tunnel syndrome caused by a medial ganglion cyst in a 56-year-old male professional golf instructor.

\section{CASE REPORT}

A 56-year-old and right-handed male patient presented with numbness and paresthesia in his right hand and fingers with duration of 3 month. Vague discomfort in his right elbow preceded a month prior to the onset of paresthesia in his right hand. At the beginning, a tingling paresthesia developed suddenly in his hypothenar and the fourth and fifth fingers when he returned home in late afternoon. In several hours, he noticed 
paresthesia with decreased sensation in those areas and mild clumsiness in his fingers. He has worked actively as a professional golf instructor for the last three decades. He denied any physical trauma or accident possibly affecting his right hand or elbow. His family and medical histories were unremarkable. Feared with sudden onset paresthesia, he was examined with magnetic resonance imaging (MRI) for the brain. No remarkable finding was found. He was initially treated with nonsteroidal anti-inflammatory drugs, physical therapy, and activity modification. However, over the next 1 month, tingling paresthesia did not improve. Clumsiness of his hand and fingers was not worsened. He was referred for further evaluation.

His neurologic examination revealed no weakness of the flexor digitorum profundus of the fourth and fifth fingers. No atrophy of intrinsic muscles of the right hand was observed. Both Froment and Wartenberg signs were negative. His sensations to light touch and pinprick were decreased in the little and ulnar aspect of the ring finger. There was no pain (spontaneously or evoked) in association with passive movement of the elbow joint. No Tinel phenomenon or tenderness overlying the ulnar nerve was observed in the cubital and Guyon tunnels. Electromyographic study demonstrated no evidence of denervation of ulnar-innervated muscle groups. However, nerve conduction study revealed a delay in sensory conduction around the elbow, indicating cubital tunnel syndrome. Because clinical and electrophysiological findings were consistent with ulnar entrapment at the elbow, an MRI of the elbow was requested.

The MRI of the right elbow showed an irregular and multilobular cyst with high signal intensity lesion on T2-weighted images (WIs) abutting to the ulnar nerve within the cubital tunnel (Fig. 1). The lesion appeared at low signal intensity on T1-WIs with rim enhancement after gadolinium (Fig. 1). The ulnar nerve was swollen and showed high signal intensity on T2-WIs. The elbow joint showed asymmetry and effusion within the joint space, tear of the cartilage of the radiohumeral joint, and osteophyte formation, indicating degenerative changes. No denervation changes were found in the flexor carpi ulnaris on T2-WIs. Laboratory examinations including erythrocyte sedimentation rate, C-reactive protein, rheumatoid factor, antinuclear antibody, and creatinine kinase were normal. Considering the course of ulnar nerve palsy and abnormal MRI findings, an exploration of the ulnar nerve within the cubital tunnel was performed.

After releasing the Osborne ligament at the cubital tunnel, the ulnar nerve was found to be swollen (Fig. 2). A multilobular cyst containing gelatinous, mucoid fluid was found to be densely adherent to the ulnar nerve was identified. The cyst was dissected carefully from the ulnar nerve under microscopic vision. A hole from tearing of the wall of ulnohumeral joint was found at the base of the cyst. We could not address any articular branch beneath the ulnar nerve at the cubital tunnel. In situ decompression of the ulnar nerve was performed.
Postoperative course was uneventful. The tingling paresthesia disappeared the next morning after operation. The clumsiness of his right hand and fingers improved within one week postoperatively. No paresthesia was felt at 6-month postoperatively. The patient's condition was stable without any recurrent symptom at follow-up of 12-month at an outpatient clinic.

\section{DISCUSSION}

\section{Pathogenesis of Ganglion Cyst in the Ulnohumeral Joint Causing Cubital Tunnel Syndrome}

Ganglion cysts are benign lesions bound by dense connective tissue filled with gelatinous fluid rich in hyaluronic acid and mucopolysaccharides ${ }^{2,12)}$. They are not line by synovium, may be unilocular or multilocular, and often have internal septa ${ }^{10)}$. They should be differentiated from synovial cysts. Synovial cysts are lined with pseudostratified columnar cells, whereas
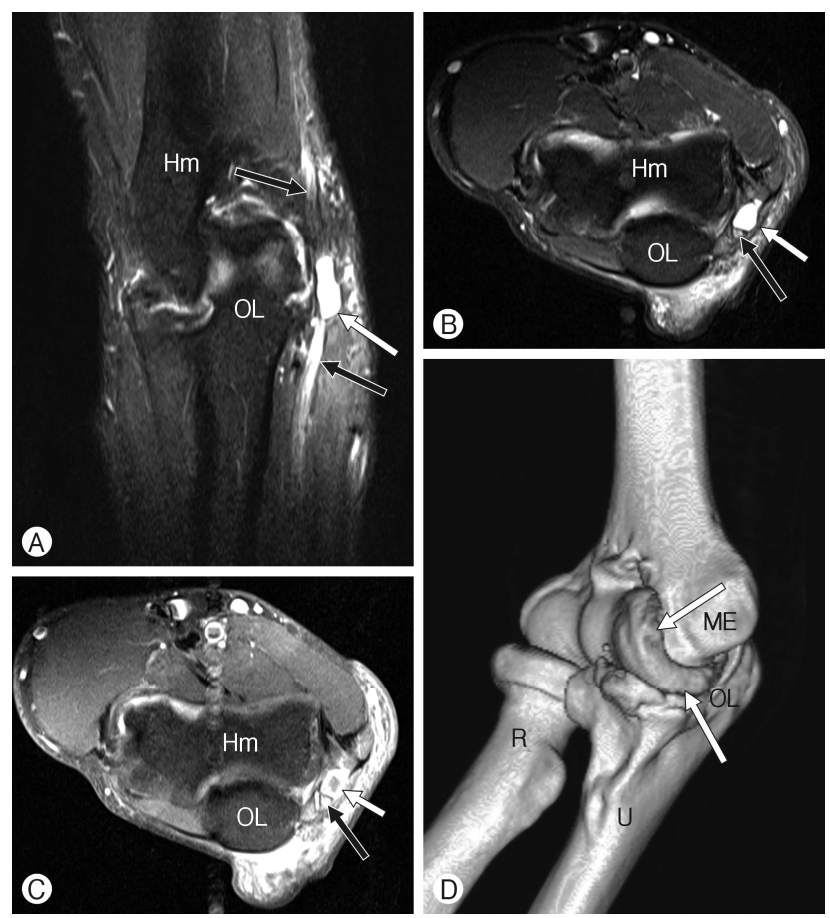

Fig. 1. Magnetic resonance imaging of ganglion cyst in the cubital tunnel syndrome. (A) T2-weighted coronal fat suppression image showing an irregularly lobulated cystic lesion (white arrow) abutting the ulnar nerve (black arrow) within the cubital tunnel. (B) T2-weighted axial fat suppression image showing the severely compressed ulnar nerve (black arrow) by a cystic lesion (white arrow). (C) T1-weighted, enhanced axial image showing a rim enhancement of the cyst within the cubital tunnel. (D) A three-dimensional computed tomographic image of the elbow joint showing marked degenerative osteophytes (arrows) in the ulnohumeral joint, indicating degenerative osteoarthritis. $\mathrm{Hm}$ : humerus; OL: olecranon; ME: medial epicondyle; R: radius; U: ulnar. 

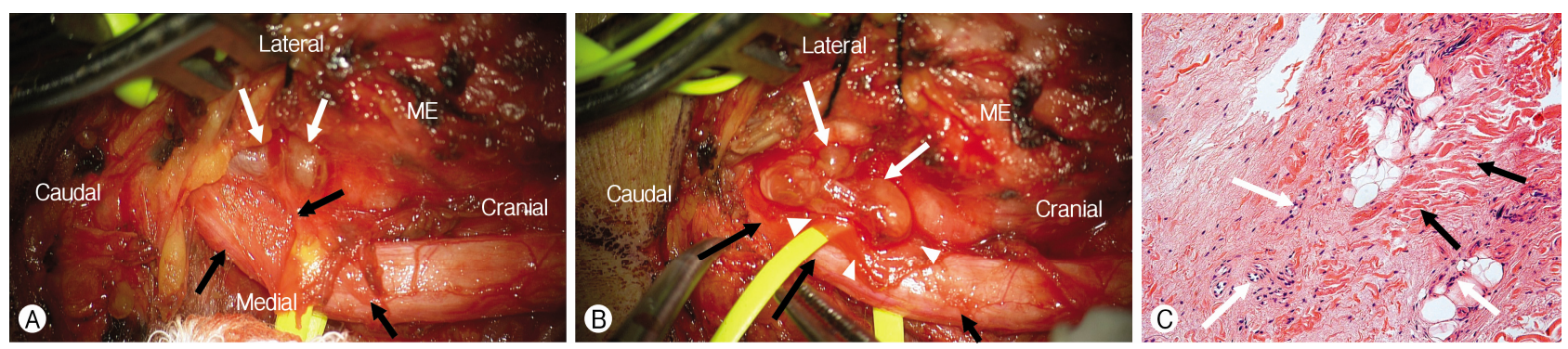

Fig. 2. Intraoperative photographs indicating the ganglion cyst compressing the ulnar nerve within the cubital tunnel. (A) An intraoperative photograph showing the compressed right ulnar nerve after opening of the right cubital tunnel. White arrows a multilobular ganglion cyst abutting the ulnar nerve. The ulnar nerve was swollen. (B) An intraoperative photograph showing gelatinous and mucoid cystic contents of the ganglion cyst (white arrows). White arrowheads indicating the dense outer layer of the ganglion cyst that produce peripheral rim enhancement on $\mathrm{Tl}$-weighted enhanced axial magnetic resonance image. (C) A histologic examination of the wall of the ganglion cyst. There are abundant fibrous tissues with keratin structure (black arrows). Many myxoid cells (white arrows) indicating marked degenerative changes are present around the glandular and adipose tissues. There was no synovial cell (hematoxylin and eosin stain [H\&E], $\times 40$ magnification). ME: medial epicondyle.

ganglion cysts have no synovial cell lining and no communication with the joint cavity ${ }^{2,7,14}$. Therefore, true differentiation between them is only possible with pathologic examinations ${ }^{7}$. In the present cases, the cyst showed communication with the joint and no synovial lining was found in the microscopic examination (Fig. 2).

Although ganglion cysts are common around the knee and wrist, those compressing the ulnar nerve in the cubital tunnel are a rare condition, ${ }^{6,13)}$. Although the incidence of symptomatic ganglion cyst causing cubital tunnel syndrome has been suggested between $1 \%$ and $8 \%{ }^{6,13)}$, this lesion has been thought to occur rarely and was not been emphasized in the literature ${ }^{6}$. Kato et al. ${ }^{6}$ reported that medial elbow ganglions were the third most common causative factor associated with cubital tunnel syndrome, with an overall prevalence of $8 \%$ (38 of 472 patients with cubital tunnel syndrome) in 2002. They suggested that ganglion cysts from medial ulnohumeral joints had a strong association with osteoarthritis of the elbow and could cause acute onset of cubital tunnel syndrome ${ }^{6}$. A patient with cubital tunnel syndrome with elbow osteoarthritis presenting medial elbow pain or severe numbness within 2 months after the onset of the syndrome should be strongly suspected of having a ganglion ${ }^{6}$. The 2 characteristic clinical symptoms of medial elbow ganglion causing cubital tunnel syndrome: intolerable medial elbow pain and a sudden onset of numbness in the ring and little finger were found in the present case. Degenerative osteoarthritic spurs at the ulnohumeral joint, reducing the size of the cubital tunnel were also found in our case.

Preoperative identification of ganglion cysts causing cubital tunnel syndrome is difficult unless MRI has been taken,13) ${ }^{6, \text { Accor- }}$ ding to Kato et al. ${ }^{6}$, 33 (87\%) of the 38 patients in their series had occult ganglion cysts that were undetected even on careful examination were only found intraoperatively. Therefore, it is suggested that MRI or ultrasound examinations should be requested for patients suspected of cubital tunnel syndrome with characteristic presentations of medial elbow pain asso- ciated with sudden onset numbness in the ring and little finger ${ }^{6}$.

\section{Surgical Techniques and Results}

Treatment of symptomatic ganglion cysts causing cubital tunnel syndrome is surgery ${ }^{6,13)}$. Regarding surgical methods of nerve decompression within the cubital tunnel, subcutaneous anterior transposition of the ulnar nerve with excision of the ganglion and adjacent joint capsule was recommended ${ }^{6,13)}$. We performed an excision of the ganglion with in-situ decompression of the ulnar nerve and could achieve an excellent outcome in 12 months in the current case. Although Kato et al.) a good outcome with relatively longer follow-up (mean 37 months) in their series, it is controversial that transposition of the ulnar nerve should be performed in all cases of ganglion cysts causing cubital tunnel syndrome.

\section{CONCLUSION}

The authors report a rare occurrence of ganglion cyst arising from medial ulnohumeral joint, causing cubital tunnel syndrome. The diagnosis of ganglion cyst within cubital tunnel was typically established with preoperative MRI examination and it was verified with intraoperative findings. Preoperative imaging studies should be performed in patients with cubital tunnel syndrome presenting with characteristic symptoms of acute onset of numbness in their ring and little fingers and medial elbow pain.

\section{CONFLICT OF INTEREST}

No potential conflict of interest relevant to this article was reported. 


\section{REFERENCES}

1. Assmus H: Simple decompression of the ulnar nerve in cubital tunnel syndrome with and without morphologic changes. Report of experiences based on 523 cases. Nervenarzt 65:846-853, 1994

2. Beaman FD, Peterson JJ: MR imaging of cysts, ganglia, and bursae about the knee. Radiol Clin North Am 45:969-982, 2007

3. Bozentka DJ: Cubital tunnel syndrome pathophysiology. Clin Orthop Relat Res 351:90-94, 1998

4. Davidge KM, Boyd KU. Ulnar nerve entrapment and injury. In: Mackinnon SE ed. Nerve surgery. New York, NY: Thieme, 2015, pp251-288

5. Gay JR, Love JG: Diagnosis and treatment of tardy paralysis of the ulnar nerve; based on a study of 100 cases. J Bone Joint Surg Am 29:1087-1097, 1947

6. Kato H, Hirayama T, Minami A, Iwasaki N, Hirachi K: Cubital tunnel syndrome associated with medial elbow Ganglia and osteoarthritis of the elbow. J Bone Joint Surg Am 84A:14131419, 2002

7. Kim J, Choi JG, Son BC: Bilateral ganglion cysts of the ligamentum flavum in the cervical spine causing a progressive cer- vical radiculomyelopathy and literature review. Case Rep Neurol Med 2017:3953641, 2017

8. Kojima T, Kurihara K, Nagano T: A study on operative findings and pathogenic factors in ulnar neuropathy at the elbow. Handchirurgie 11:99-104, 1979

9. Ochiai N, Honmo J, Tsujino A, Nisiura Y: Electrodiagnosis in entrapment neuropathy by the arcade of Struthers. Clin Orthop Relat Res 378:129-135, 2000

10. Perdikakis E, Skiadas V: MRI characteristics of cysts and "cystlike" lesions in and around the knee: what the radiologist needs to know. Insights Imaging 4:257-272, 2013

11. Sheldon WD: Tardy paralysis of the ulnar nerve. Med Clin North Am 5:499-509, 1921

12. Tom BM, Rao VM, Farole A: Bilateral temporomandibular joint ganglion cysts: CT and MR characteristics. AJNR Am J Neuroradiol 11:746-748, 1990

13. Tong J, Xu B, Dong Z, Liu J, Zhang C, Gu Y: Cubital tunnel syndrome caused by ganglion cysts: a review of 59 cases. Acta Neurochir (Wien) 159:1265-1271, 2017

14. Weiss SW, Goldblum JR. Benign tumors and tumor-like lesions of synovial tissue. In: Weiss SW, Goldblum JR eds. Enzinger and Weiss's soft tissue tumors, ed 4th. St. Louis, MO: Mosby, 2001, pp1037-1062 